\title{
Classification of Turbulent Diffusions in the Atmosphere*
}

\author{
Y. Ogura, Y. Sekiguchi** and K. Miyakoda \\ Geophysical Institute, Tokyo University \\ (Manuscript received 26 June, 1958)
}

\section{Contents}

1. Introduction

$\S 2$. The instantaneous source type and the continuous fixed source type in a fluid flow.

$\$ 3$. The fixed continuous source type in a fluid at rest.

$\S 4$. The continuous floating source type.

$\$ 5$. Turbulent Diffusion from a body source.

$\S 6$. The interrelation between the Eulerian and Lagrangian correlation functions.

\section{- Abstract -}

Turbulent diffusions of matter (gas, minute particles or heat) emitted from a point source are classified into the following four types, and interrelations among them are discussed: (1) the instantaneous source type, (2) the continuous fixed source type in a fluid flow, (3) the continuous fixed source type in a fluid at rest (4) the continuous floating source type.

As an extension of this research, the turbulent diffusion of matter from a body source in an isotropic turbulent field is discussed in the latter part of this paper. Furthermore, some remarks are made on the interrelation between the so-called Eulerian and Lagrangian correlation functions, and the equation expressing the wind velocity profile near the ground is derived by making use of the Lagrangian correlation.

\section{§ 1. Introduction}

In the preceding papers, one of the present authors has theoretically studied the turbulent diffusion of matter (gas, minute particles or heat) emitted continuously from a fixed point source in a homogeneous and isotropic turbulent field (Ogura, $1952 \mathrm{a}, \mathrm{b}, \mathrm{c}$ ). In order to have the complete informations concerning this subject, however, it is necessary to research a few other types of turbulent diffusion, even if we confine ourselves to the case of dispersion of matter emitted from a point source, because the features of the dispersion of matter are widely different from each other, according to conditions under which the matter is dispersed. The factors which distinguish these types seem to be as follows:

(i) Either the source emits the matter continuously or not. In the latter case,

* Division of Meteorology, Contribution No. 63

** Present affiliation: Central Meteorological Observatory, Tokyo 
the matter is concentrated at an origin at the initial time and then begins to disperse. The names, continuous and instantaneous sources, are used for sources in the former and latter cases, respectively.

(ii) Either the source is fixed to the earth or not. The source in the latter case is floating in a fluid and moves to and fro with the wind. The names, fixed and floating sources, are adequate for sources in the former and latter cases, respectively.

(III) Either the source is placed in a fluid flow or in a fluid at rest, or in other words, either there exists a general flow or not. In this sentence, the meaning of general flow must be carefully used in the problem of turbulent diffusion. It is obvious that the case in which the source is fixed and there is no general flow belongs to the latter case, but it is reasonably considered that the case in which a general flow exists and the source itself is moving with the same velocity with that of the general flow should belongs also to the latter case. Because the general flow has no influence on the dispersion of the matter in this case. In a similar fashion, the cases in which the source is fixed and a general flow exists or the source is moving with a constant velocity but different from that of the general flow belong to the former case.

Then, the combination of these three factors should produce 8 kinds of turbulent diffusion types. Among them, however, some types are exactly the same with each other and some types cannot appear in practice. For instance, the continuous floating source type in a fluid flow is the same with that in a fluid at rest, and the instantaneous fixed source type can not appear. Furthermore, it is evident that the diffusion of the instantaneous source types are the same, irrespective of the existence of the general flow.

From these considerations, it may be concluded that the kinds of turbulent diffusions are classified into the following four types.

(1) the continuous fixed source type in a fluid flow,

(2) the continuous fixed source type in a fluid at rest,

(3) the continuous floating source type,

(4) the instantaneous source type.

One of the purposes of the present paper is to discuss the turbulent diffusions of these four types in detail and to study the interrelation among these diffusion phenomena.

\section{§ 2. The instantaneous source type and the continuous fixed source type in a fluid flow.}

For simplification, we consider the diffusion of the matter only in the direction $y$, perpendicular to the direction of mean flow. Let us now consider one of the clouds of particles which is concentrated at the initial time and at the origin of diffusion. These particles will be dispersed in twofold manners, i.e., the dispersion of the center of the gravity of this cloud, and the diffusion of particles around this center, as described 
by Inoue (1952). Let $P\left(0, y_{g} ; 0, \varphi\right) d y_{g}$ denote the probability that a center of the gravity of this cloud is to be found in the interval between $y_{g}$ and $y_{\sigma}+d y_{g}$ after the time $\varphi$, and let $W\left(y-y_{\theta}, \varphi\right) d y$ denote the probability that a particle is to be found at a distance $y-y_{g}$ from the center, when the position of the center is $y_{a}$. Then the probability that a particle is to be found in the interval between $y$ and $y+d y$ after the time $\varphi$ is given by

$$
W_{\Gamma}(y, \varphi) d y=\int_{-\infty}^{\infty} P\left(0, y_{g} ; 0, \varphi\right) W\left(y-y_{g}, \varphi\right) d y_{g} d y
$$

and the standard deviation of the positions of the particles is

$$
\left\langle Y^{2}\right\rangle_{\mathrm{I}}=\int_{-\infty}^{\infty} y^{2} W_{I}(y, \varphi) d y
$$

Although this spreading of particles is obtained as the overlapping mean of the clouds of particles emitted instantaneously from the origin, it is obvious that this is nothing but the spreading in the case of the continuous fixed source type in a fluid flow. Because the word "continuous source" means the source which emits continuously a number of clouds at every instant and the clouds thus emitted are overlapped at the stage of the same process time $\varphi$ at a point $x=U \varphi$, where $U$ represents the mean velocity. Owing to this circumstance, the continuous fixed source type in a fluid flow may be reasonably called the overlapped instantaneous source type*.

The spreading of particles in the case of the instantaneous source type is given by

$$
\left\langle Y^{2}\right\rangle_{\mathrm{N}}=\int_{-\infty}^{\infty} y^{2} W\left(y-y_{g}, \varphi\right) d y
$$

As can be seen easily from (2.2) and (2.3), the difference between the diffusion of overlapped instantanous source type and that of instantaneous source type is as follows: although the spreading of the center of the cloud is taken into consideration in the former case, the spreading in the latter is measured always from the center of the cloud.

One of the present authors previously discussed the spreading $\left\langle Y^{2}\right\rangle_{\mathrm{f}}$, taking into consideration the effect of the observational time (or length of the sampling period) on the concentration of the particles, and the following results were obtained (1952 c).

$$
\begin{aligned}
\left\langle Y^{2}(t)\right\rangle_{\mathrm{I}} & =\left\langle V^{2}\right\rangle_{T * t^{2}}\left(1-\frac{t}{3 \tau_{0 T *}}\right), \quad 0 \leqq t \leqq \tau_{0 T *}, \\
& =\left\langle V^{2}>_{T *} \tau_{0} T *\left(t-\frac{1}{3} \tau_{0 T *}\right), \quad t \geq \tau_{0 T *},\right.
\end{aligned}
$$
follows:

${ }^{*}\left\langle Y^{2}\right\rangle_{1}$ in $(2.2)$ is expressed by means of the Lagrangian correlation function $R(\xi)$ as

$$
\left\langle Y^{2}\right\rangle_{\mathrm{I}}=2\left\langle V^{2}\right\rangle \int_{0}^{t} \int_{0}^{\eta} R(\xi) d \xi d \eta
$$

In Frenkiel's paper (1952 a), this type of diffusion is called the dispersion from an instantaneous point source and Inoue (1952) called this the fixed source type. 
where $\left\langle V^{2}\right\rangle_{T_{*}}$ denotes the energy of turbulence effective for diffusion during the sampling period $T_{*}$, and the relation between $\left\langle V^{2}\right\rangle_{*_{*}}$ and $T_{*}$ is

$$
\begin{aligned}
<V^{2}>_{T *} & =\left\langle V^{2}>_{\infty} \frac{T_{*}}{\tau_{0}},\right. & & T_{*} \leq \tau_{0}, \\
& =<V^{2}>_{\infty}, & & T_{*} \leq \tau_{0} .
\end{aligned}
$$

Here, $\quad \tau_{0}$ : the life time of the largest turbulent element, $\left\langle V^{2}\right\rangle_{\infty}$ : the total energy of turbulence.

The equation (2.5) expresses that turbulent elements which contribute to the turbulent diffusion during the sampling period $T_{*}$ are by no means all of the turbulent elements existing in that turbulent field, but only those whose life time are smaller than $T_{*}$. Furthermore, $\tau_{0 T *}$ denotes the life time of the effective largest turbulent element for the length of sampling period $T_{*}$, and is given by

$$
\begin{aligned}
\tau_{0} T_{*} & =T_{*}, & & T_{*} \leq \tau_{0}, \\
& =\tau_{0}, & & T_{* \geq \tau_{0},},
\end{aligned}
$$

i.e., the life time of the effective largest turbulent element increases in proportion to $T_{*}$ so far as $T_{*}$ is smaller than the life time of the largest turbulent element.

The spreading of particles in the case of instantaneous source is obtained by replacing $T_{*}$ in (2.4) by $t$, i. $e_{\text {., }}$

or

$$
\begin{aligned}
<Y^{2}>_{\pi} & =\frac{2}{3}<V^{2}>_{\infty} \frac{t^{3}}{\tau_{0}} \quad t \leqq \tau_{0}, \\
& =<V^{2}>_{\infty} \tau_{0}\left(t-\frac{1}{3} \cdot \tau_{0}\right), \quad t \geq \tau_{0},
\end{aligned}
$$

$$
\begin{aligned}
\left\langle Y^{2}>_{\mathrm{rV}} / L_{0}{ }^{2}\right. & =\frac{2}{3}\left(t / \tau_{0}\right)^{3}, & & t \leqq \tau_{0}, \\
& =\frac{t}{\tau_{0}}-\frac{1}{3} . & & t \supseteqq \tau_{0},
\end{aligned}
$$

where $L_{0}=\left\langle V^{2}\right\rangle^{1 / 2} \cdot \tau_{0}$ means the scale of the largest turbulent element. The result that $\left\langle Y^{2}\right\rangle_{\text {rv }}$ increases in proportion to $t^{3}$ was already derived by several authors from various points of view (Batchelor 1950, Inoue 1951, Ichiye 1951, Ogura 1952 b). (cf. Fig. 1.)

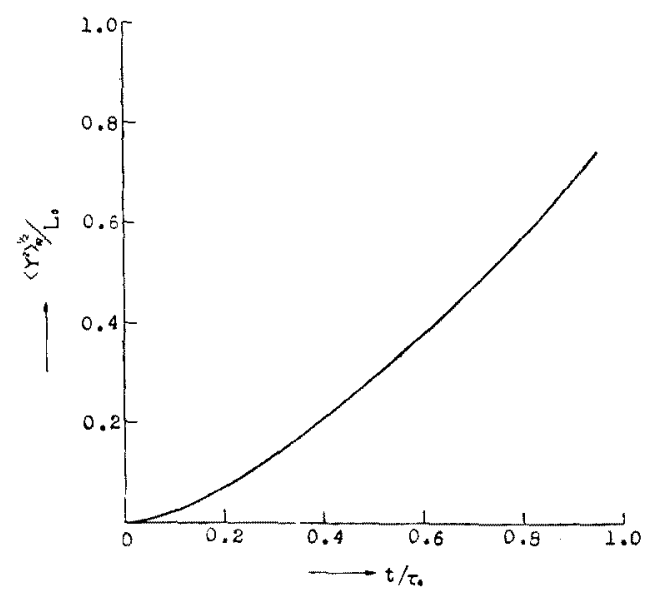

Fig. 1

\section{§ 3. The fixed continuous source type in a fluid at rest.}

The difference between the turbulent diffusion of continuous fixed source type in a fluid at rest and that in a fluid flow is as follows: the diffused cloud should be overlapped 
at the same diffusion stage (i.e., at the same time $t$ ) in the latter case and at the other diffusion stage in the former.

Therefore, if we denote the number of particles which are emitted from the source during the time interval between $\varphi$ and $\varphi+d \varphi$ with $s(\varphi) d \varphi$, the probability that a particle is to be found between $y$ and $y+d y$ at the time $t$ is given by

$$
W_{\mathrm{II}}(y, t)=\frac{1}{N} \int_{0}^{t} s(t-\varphi) \int_{-\infty}^{\infty} P\left(0, y_{g} ; t-\varphi, t\right) W\left(y-y_{g}, \varphi\right) d y_{\sigma} d \varphi
$$

where

$$
N=\int_{t}^{0} s(t-\varphi) d \varphi,
$$

By transforming (3.1) slightly, we get easily

$$
W_{\mathrm{II}}(y, t)=\frac{1}{N} \int_{0}^{t} s(\eta) W_{\mathrm{I}}(y, t-\eta) d \eta
$$

where

$$
\eta=t-\varphi \text {. }
$$

When the emission rate of the source is independent of time and $q$ denotes the number of particles emitted per unit time,

$$
N=q t,
$$

and (3.3) goes over to

$$
W_{\mathrm{II}}=\frac{1}{t} \int_{0}^{t} W_{1}(y, t-\eta) d \eta=\frac{1}{t} \int_{0}^{t} W_{\mathrm{I}}(y, \zeta) d \zeta .
$$

In this case, the spreading of the particles is given by

$$
\begin{aligned}
\left\langle Y^{2}\right\rangle_{\mathrm{I}} & =\int_{-\infty}^{\infty} y^{2} W_{\mathrm{II}}(y, t) d y \\
& =\frac{1}{t} \int_{-\infty}^{\infty} \int_{0}^{t} y^{2} W_{\mathrm{I}}(y, \zeta) d \zeta d y=\frac{1}{t} \int_{0}^{t}\left\langle Y^{2}(\zeta)>_{\mathrm{I}} d \zeta,\right.
\end{aligned}
$$

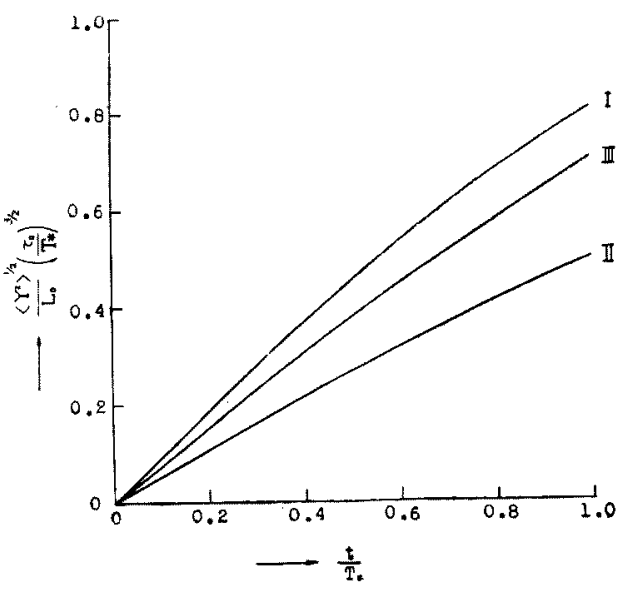

Fig. 2

or in words, $\left\langle Y^{2}\right\rangle_{\mathrm{II}}$ is equal to the average of $\left\langle Y^{2}\right\rangle_{\mathrm{I}}$ for the interval $t$. When $\left\langle Y^{2}(t)\right\rangle_{\mathrm{I}}$ is given by $(2.4),\left\langle Y^{2}(t)\right\rangle_{\mathrm{II}}$ is given as follows:

$$
\left\langle Y^{2}>_{\mathrm{II}}=\frac{\left\langle V^{2}\right\rangle_{T *}}{3} t^{2}\left(1-\frac{t}{4 \tau_{0 T *}}\right)\right. \text {. }
$$

The functional form of (3.5) is shown in Fig. 2 with $\left\langle Y^{2}\right\rangle_{\mathrm{I}}$.

\section{$\S 4$. The continuous floating source.}

When the point source is not fixed to the earth, the source itself moves with the wind. In the case of the turbulent diffusion of continuous floating source with (or 
without) general flow type, therefore, the influence of the random motion of the source itself must be taken into account. When $P_{s}\left(0, y_{s} ; 0, \eta\right)$ denotes the probability that the source is to be found between $y_{s}$ and $y_{s}+d y_{s}$ at the time $\eta$, the probability of a particle $W_{\mathrm{mI}}(y, t)$ is given by

$$
\begin{aligned}
W_{\mathrm{III}}(y, t)= & \frac{1}{N} \int_{0}^{t} s(\eta) \int_{-\infty}^{\infty} P_{s}\left(0, y_{s} ; 0, \eta\right) \int_{-\infty}^{\infty} P\left(y_{s}, y_{g} ; \eta, t\right) \\
& W\left(y-y_{g}, t-\eta\right) d y_{g} d y_{s} d \eta
\end{aligned}
$$

When the scale of the source is so small that we may regard its motion as simlar to that of emitted minute particles, it is reasonably assumed that

$$
P_{s}\left(0, y_{s} ; 0, \eta\right)=W_{\mathrm{f}}\left(y_{s}, \eta\right)
$$

Noting that

$$
\int_{-\infty}^{\infty} P\left(y_{s}, y_{g} ; \eta, t-\eta\right) W\left(y-y_{g}, t-\eta\right) d y_{s}=W_{\mathrm{I}}\left(y-y_{s}, t-\eta\right),
$$

we can transform (4.1) to

$$
\left.W_{\mathrm{II}}(y, t)=\frac{1}{N} \int_{0}^{t} \int_{-\infty}^{\infty} s(\eta) W_{\mathrm{I}}^{\prime} y_{s}, \eta\right) W_{\mathrm{I}}\left(y-y_{s}, t-\eta\right) d y_{s} d \eta
$$

When $s(\eta)$ is independent of time, (4.3) is reduced to

$$
W_{\mathrm{TII}}(y, t)=\frac{1}{t} \int_{0}^{t} \int_{-\infty}^{\infty} W_{\mathrm{I}}\left(y_{s}, \eta\right) W_{\mathrm{I}}\left(y-y_{s}, t-\eta\right) d y_{s} d \eta
$$

To make clear the difference between $W_{\mathrm{III}}(y, t)$ and $W_{\mathrm{II}}(y, t)$, we shall now assume that $W_{\mathrm{I}}$ has the following functional forms:

$$
W_{\mathrm{I}}\left(y_{s}, \eta\right)=\frac{1}{\sqrt{a \pi}} e^{-\frac{y_{s}^{2}}{a}} \quad W_{\mathrm{T}}\left(y-y_{s}, t-\eta\right)=\frac{1}{\sqrt{b \pi}} e^{-\frac{\left(y-y_{s}\right)^{2}}{b}},
$$

It is then easily shown that

$$
\int_{-\infty}^{\infty} W_{\mathrm{I}}\left(y_{s}, t\right) W_{\mathrm{r}}\left(y-y_{s}, t-\eta\right) d y_{s}=\frac{1}{\sqrt{\pi(a+b)}} e^{-\frac{y^{2}}{a+b}}
$$

Therefore, we get from (4.3)

$$
W_{\mathrm{III}}(y, t)=\frac{1}{\sqrt{\pi} N} \int_{0}^{l} s(\eta) \frac{1}{\sqrt{a+b}} e^{-\frac{y^{2}}{a+b}} d \eta
$$

On the other hand, $W_{\mathrm{IJ}}(y, t)$ is

$$
W_{\mathrm{rr}}(y, t)=\frac{1}{\sqrt{\pi} N} \int_{0}^{t} s(\eta) \frac{1}{\sqrt{b}} e^{-\frac{y^{2}}{b}} d \eta
$$

provided $W_{\mathrm{I}}$ is given by (4.5).

The spreadings of particles are 
and

$$
\begin{aligned}
\left\langle Y^{2}(t)\right\rangle_{\mathrm{n}} & =\frac{1}{\sqrt{\pi} N} \int_{0}^{t} \frac{s(\eta)}{\sqrt{b}} \int_{-\infty}^{\infty} y^{2} e^{-\frac{y^{2}}{b}} d y d \eta=\frac{1}{2 N} \int_{0}^{t} b s(\eta) d \eta \\
& =\frac{1}{N} \int_{0}^{t} s(\eta)\left\langle Y^{2}(t-\eta)>_{\mathrm{I}} d \eta\right.
\end{aligned}
$$

Noting that

$$
\begin{aligned}
\left\langle Y^{2}\right\rangle_{\mathrm{II}} & =\frac{1}{\sqrt{\pi} N} \int_{0}^{t} \frac{s(\eta)}{\sqrt{a+b}} \int_{-\infty}^{\infty} y^{2} e^{-\frac{y^{2}}{a+b}} d y d \eta=\frac{1}{2 N} \int_{0}^{t} s(\eta)(a+b) d \eta \\
& =\frac{1}{N} \int_{0}^{t} s(\eta)\left\{\left\langle Y^{2}(\eta)\right\rangle_{\mathrm{I}}+\left\langle Y^{2}(t-\eta)\right\rangle_{\mathrm{I}}\right\} d \eta .
\end{aligned}
$$

$$
\int_{0}^{t}\left\langle Y^{2}(\eta)\right\rangle_{1} d \eta=\int_{0}^{t}\left\langle Y^{2}(t-\eta)\right\rangle_{1} d \eta
$$

the spreading $\left\langle Y^{3}\right\rangle_{\mathrm{Ir}}$ in the case of constant emission rate is simply given by

$$
\left\langle Y^{2}(t)\right\rangle_{\mathrm{III}}=\frac{2}{t} \int_{0}^{t}\left\langle Y^{r_{2}}(\eta)\right\rangle_{\mathrm{I}} d \eta=2\left\langle Y^{2}(t)\right\rangle_{\mathrm{II}}
$$

When $\left\langle Y^{2}(t)\right\rangle_{\mathrm{II}}$ is given by (3.5), $\left\langle Y^{2}(t)\right\rangle_{\mathrm{III}}$ is expressed as follows:

$$
\left\langle Y^{\mathrm{u}}(t)>_{\mathrm{H} L}=\frac{2}{3}<V^{2}\right\rangle_{\infty} t^{2}\left(1-\frac{t}{4 \tau_{0 \tau_{*}}}\right)
$$

The shape of this spreading is also shown in Fig. 2.

\section{\$ 5. Turbulent Diffusion from a body source}

We have described so far the spreading of clouds of particles from a point source by means of the variance $\left\langle Y^{2}\right\rangle$. We shall now consider the case of the turbulent diffusion from a body source and the change of the concentration of particles.

At first, let us consider one of the clouds of particles which are concentrated at the initial time at $(x, y, z)$. These particles will be then distributed in the cloud, following a law of probability that a particle is to be found at a point $\left(x^{\prime}, y^{\prime}, z^{\prime}\right.$,). We shall assume this prabability is given by

$$
W_{\mathrm{I}}\left(x, y, z ; x^{\prime}, y^{\prime}: z^{\prime} ; 0, t\right)=\frac{1}{\left(2 \pi<Y^{2}>\right)^{3 / 2}} \exp \left[-\frac{1}{2<Y^{2}>}\left\{\left(x^{\prime}-x\right)^{2}+\left(y^{\prime}-y\right)^{2}+\left(z^{\prime}-z\right)^{2}\right\}\right]
$$

In this representation, the variance $\left\langle Y^{2}\right\rangle$ is a function of time and is expressed by the equation (2.4).

Then, if the concentration of clouds of particles per unit volume of fluid at the initial time and at a point $(x, y, z)$ is $s(x, y, z, 0)$, the concentration at the time $t$ and at a point $\left(x^{\prime}, y^{\prime}, z^{\prime}\right)$ is given by

$$
\begin{gathered}
s\left(x^{\prime}, y^{\prime}, z^{\prime} ; t\right)=\frac{1}{\left(2 \pi<Y^{2}>\right)^{3 / 2}} \iiint s(x, y, z ; 0) \exp \left[-\frac{1}{2<Y^{2}>}\left\{\left(x^{\prime}-x\right)^{2}\right.\right. \\
\left.\left.+\left(y^{\prime}-y\right)^{2}+\left(z^{\prime}-z\right)^{2}\right\}\right] d x d y d z \\
-7-
\end{gathered}
$$


where integration with respect to $(x, y, z)$ should be done all over the whole domain in which $s(x, y, z, 0)$ differs from zero.

By this equation $(5: 1)$, the change of the concentration of particles with time is represented.

(i) Consider now the case in which $t$ is small, compared with $\tau_{0}$. In this case, $s(x, y, z, 0)$ can be expanded as follows:

$$
s(x, y, z ; 0)=s\left(x^{\prime}, y^{\prime} \cdot z^{\prime} ; 0\right)+\left(x^{\prime}-x\right) \frac{\partial s}{\partial x}+\ldots+\frac{\left(x^{\prime}-x\right)^{2}}{2 !} \frac{\partial^{2} s}{\partial x^{2}}+\ldots
$$

Substituting (5.2) into (5.1), we have

$$
s\left(x^{\prime \prime}, y^{\prime}, z^{\prime} ; t\right)=s\left(x^{\prime}, y^{\prime}, z^{\prime} ; 0\right)+\frac{\leq Y^{\prime} \geq}{2 !}\left\{\frac{\partial^{2} s}{\partial x^{2}}+\frac{\partial^{2} s}{\partial y^{2}}+\frac{\partial^{2} s}{\partial z^{2}}\right\}_{x^{\prime}, y^{\prime}, z^{\prime}, t=0}^{+}
$$

On the other hand, Proudmann (1948) obtained a similar result in his research on the diffusion of salinity in the ocean. His result is expressed, in our notations, as follows:

$$
\begin{aligned}
s\left(x^{\prime}, y^{\prime}, z^{\prime} ; t\right) & =\left\{1+\frac{1}{2 !}\left(\left\langle X^{2}\right\rangle \frac{\partial^{2}}{\partial x^{2}}+\left\langle Y^{2}\right\rangle \frac{\partial^{2}}{\partial y^{2}}+\left\langle Z^{2}\right\rangle \frac{\partial^{2}}{\partial z^{2}}\right)\right. \\
& +\left\langle X Y>\frac{\partial^{2}}{\partial x \partial y}+\ldots\right\} s(x, y, z ; 0) .
\end{aligned}
$$

When the turbulence is isotropic, we get

$$
\left\langle X^{2}\right\rangle=\left\langle Y^{2}\right\rangle=\left\langle Z^{2}\right\rangle
$$

and

$$
\langle X Y\rangle=\langle X Z\rangle=\ldots \ldots \ldots=0,
$$

as is shown in another paper (Ogura and Miyakoda, 1953). Then, (5.4) is reduced to

$$
s\left(x^{\prime}, y^{\prime} z^{\prime}, t\right)=s\left(x^{\prime}, y_{y}^{\prime} z^{\prime}, 0\right)+<Y^{2} \geq\left\{\begin{array}{l}
\partial^{2} s \\
\partial x^{2}
\end{array}+\frac{\partial^{2} s}{\partial y^{2}}+\frac{\partial^{2} s}{\partial z^{2}}\right\}+\ldots .
$$

This coincides with our result (5.3).

(ii) When $t$ is large, $\left\langle Y^{2}\right\rangle$ is represented by

$$
\left\langle Y^{2}\right\rangle=\left\langle V^{2}\right\rangle_{\infty} \tau_{0} t
$$

Then, (5.1) becomes as follows:

$$
\begin{aligned}
s\left(x^{\prime}, y^{\prime}, z^{\prime} ; t\right) & =\frac{1}{\left(2 \pi<V^{2}>\tau_{0} t\right)^{3 / 2}} \iiint s(x, y, z, 0) \exp \left[-\frac{1}{2<V^{2}>\tau_{0} t}\right. \\
& \left.\times\left\{\left(x^{\prime}-x\right)^{2}+\left(y^{\prime}-y\right)^{3}+\left(z^{\prime}-z\right)^{2}\right\}\right] d x d y d z .
\end{aligned}
$$

A simple calculation shows that this $s\left(x^{\prime}, y^{\prime}, z^{\prime}, t\right)$ satisfies the following equation of diffusion:

$$
\begin{gathered}
\frac{\partial s}{\partial t}=\frac{\left\langle V^{2}>\tau_{0}\right.}{2}\left(\frac{\partial^{2} s}{\partial x^{\prime 2}}+\frac{\partial^{2} s}{\partial y^{\prime 2}}+\frac{\partial^{2} s}{\partial z^{\prime 2}}\right) \\
-8-
\end{gathered}
$$


These results show that the solution of the differential equation of diffusion with constant diffusivity $\frac{1}{2}\left\langle V^{2}>\tau_{0}\right.$ can precisely describe the change of the concentration only when $t$ is very large compared with $\tau_{0}$, as was already pointed out by Frenkiel (1952 b).

To illustrate this point, a numerical example is presented for the case of instantaneous source type. The analytical solution of $(5.6)$ for this case is

where

$$
s\left(x^{\prime} \quad y^{\prime}, z^{\prime} ; t\right)=\frac{s_{0}}{(4 \pi K t)^{3 / 2}} \exp \left(-\frac{r^{2}}{4 K f}\right)
$$

$$
K=\frac{1}{2}<V^{2}>\tau_{0}, \quad r=\left(x^{\prime 2}+y^{\prime 3}+z^{\prime 2}\right)^{1 / 2} .
$$

On the other hand, taking account of the microstructure of the turbulence, the solution which is derived from $(5.1)$ becomes

$$
s\left(x^{\prime}, y^{\prime}, z^{\prime} ; t\right)=\frac{s_{0}}{\left(2 \pi<Y^{2}>\right)^{3 / 2}} \exp \left(-\frac{r^{2}}{2<r^{2}>}\right)
$$

When $t \gg \tau_{0},\left\langle Y^{2}\right\rangle=\left\langle V^{2}\right\rangle \tau_{0} t$. Therefore, (5.8) is reduced in this case to

$$
\begin{aligned}
s\left(x^{\prime}, y^{\prime}, z^{\prime} ; t\right) & =\frac{s_{0}}{\left(2 \pi<V^{2}>\tau_{0} t\right)^{3 / 2}} \exp \left\{-\frac{r^{2}}{2<V^{2}>\tau_{0} t}\right\} \\
& =\frac{s_{0}}{(4 \pi K t)^{3 / 2}} \exp \left(-\frac{r^{2}}{4 K t}\right)
\end{aligned}
$$

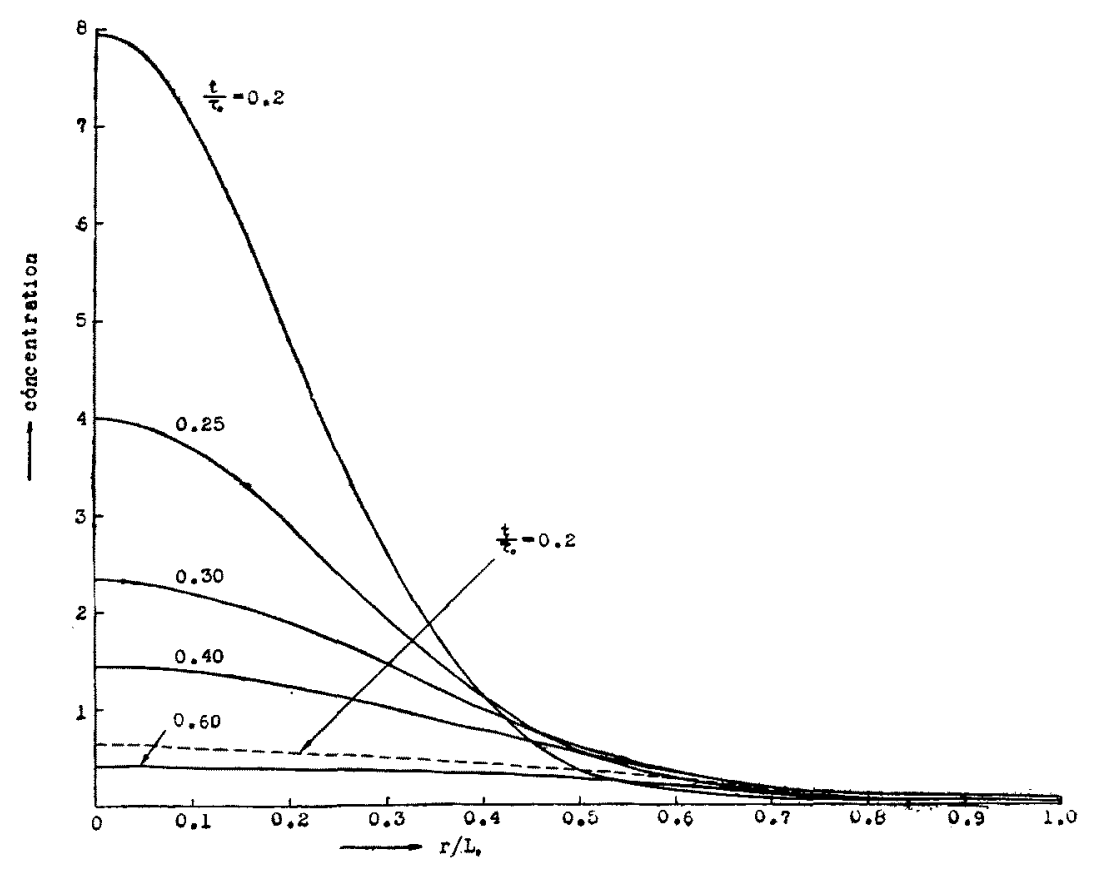

Fig. 3 
and this is exactly the same with $(5 \cdot 7)$. Full lines in Fig. 3 show the numerically calculated results of (5.8), $s$ being expressed in an arbitrary unit, and dashed lines show those of (5.7). As is seen in this figure, the solutions of the equation represent a too much rapid decrease in concentration near the center.

\section{$\S$ 6. The interrelation between the Eulerian and Lagrangian correlation functions.}

As is well known, three different kinds of correlation coefficients play an important rôle in the studies both of the structure of isotropic turbulent field and of the turbulent diffusion in this field. They are the so-called Eulerian- $\left(\boldsymbol{R}_{E}(t)\right)$, Taylor-Kármánian $\left(\boldsymbol{R}_{T_{K}}(x)\right)$ and Lagrangian $\left(R_{L}(\xi)\right)$ correlation coefficients. $R_{E}(t)$ is the auto-correlation coefficient between turbulent wind velocities observed at a fixed point, and $R_{T K}(x)$ is that between velocities observed at two points separated by the distançe $x$ at the same time, and finally $R_{L}(\xi)$ is that between velocities of a particles floating in the turbulent field at a certain time and at the time after an interval of time $\xi$.

Among these correlations, it may be reasonably considered that the functional forms of $R_{E}(t)$ and $R_{T K}(x)$ coincide with each other, when the turblent wind velocities are sufficiently small, compared to the mean flow velocity, i.e., if all turbulent elements flow along the $x$-axis with the mean velocity $U$, it may be permissible to derive $\boldsymbol{R}_{T^{K}}(x)$ from $\boldsymbol{R}_{E}(t)$ by replacing $t$ in the latter with $x / U$.

We shall then deal with the relationship between $R_{B}(t)$ and $R_{L}(\xi)$ in this section somewhat more generally than before (Ogura, 1952 a).

As was shown by Sir Geoffrey Taylor (1921), the diffusion width is given by means of the Lagrangian correlation as follows:

$$
\left\langle Y^{2}\right\rangle=2<V^{2}>\int_{0}^{t} \int_{0}^{\eta} R_{i}(\xi) d \xi d \eta
$$

or

$$
<Y^{2}>=2<V^{2}>\int_{0}^{t}(t-\xi) R_{L}(\xi) d \xi
$$

as shown by J. Kampe de Feriet (1939) and F. N. Frenkiel (1949). On the other hand, $\left\langle Y^{2}\right\rangle$ can be also expressed by means of $R_{T K}(x)$ as follows (Ogura, 1952 a):

$$
\left\langle Y^{2}\right\rangle=\frac{\left.2 t^{2}<V^{2}\right\rangle}{\delta^{2}} \int_{0}^{\delta}(\delta-x) R_{T K}(x) d x,
$$

where $t$ represents the time which elapses since a certain particle has been emitted from the point source. In the same meaning, the name "process time" was used by E. Inoue (1952) and Y. Ogura (1952) and "dispersion time" was used by F. N. Frenkiel (1552 a). $\delta$ in (6.2) is a function of $t$ and the relation between $t$ and $\delta$ is given by the equation: 


$$
\delta=t<V^{2}>^{1 / 2}\left[1-\frac{2}{\delta^{2}} \int_{0}^{\delta}(\delta-x) R_{T K}(x) d x\right]
$$

From (6.1) and (6.2) we get the following relation between $\boldsymbol{R}_{L}(t)$ and $\boldsymbol{R}_{T K}(x)$ :

or

$$
R_{L}(t)=\frac{1}{\left.2<V^{2}\right\rangle} \frac{d^{2}<Y^{2}>}{d t^{2}}=\frac{d^{2}}{d t^{2}}\left[\frac{t^{2}}{\delta^{2}} \int_{0}^{\delta}(\delta-x) R_{T_{K}}(x) d x\right]
$$

$$
\frac{1}{\delta^{2}} \int_{0}^{\delta}(\delta-x) R_{T K}(x) d x=\frac{1}{t^{2}} \int_{0}^{t}(t-\xi) R_{L}(\xi) d \xi
$$

If we introduce other variables $\eta$ and $\zeta$ by the respective relations of

$$
\frac{x}{\delta}=\eta, \quad \frac{\xi}{t}=\zeta,
$$

$(6.4)^{\prime}$ is rewritten in a very symmetrical form;

$$
\int_{0}^{1}(1-\eta) R_{T K}(\eta) d \eta=\int_{0}^{1}(1-\zeta) R_{L}(\zeta) d \zeta
$$

By the equations $(6.4)$ or $(6.4)^{\prime \prime}$ and $(6.2), \boldsymbol{R}_{L}(t)$ is related to $\boldsymbol{R}_{T_{K}}(x)$ or $\boldsymbol{R}_{E}(t)$. For example, let $\boldsymbol{R}_{T K}(x)$ be given by

$$
\begin{aligned}
\boldsymbol{R}_{T K}(x) & =1-\left(\frac{x}{x_{0}}\right)^{m} & & x \leqq x_{0}, \\
& =0, & & x \geqq x_{0},
\end{aligned}
$$

where $x_{0}$ is the characteristic length of the turbulent field and means the scale of the largest turbulent element in that turbulent field. Then, $R_{n}(t)$ is

$$
\begin{aligned}
\boldsymbol{R}_{E}(t) & =1-\left(\frac{t}{t_{0}}\right)^{m}, & & t \leqq t_{0}, \\
& =0, & & t \geqq t_{0},
\end{aligned}
$$

where $t_{0}\left(=x_{0} / U\right)$ means the time interval during which the largest turbulent element passes through the observation point and the name "passage time" of the largest turbulent element may be appropriate for this characteristic period. (E. Inoue, 1950.)

Substituting (6.6) into (6.3), we get

$$
\begin{aligned}
\delta & =\left[t^{2}\left\langle V^{2}>\frac{2}{x_{0}^{m}(m+1)(m+2)}\right]^{\frac{1}{2-m},} \quad \delta \leqq x_{0},\right. \\
& \left.=t<V^{2}\right\rangle^{1 / 2}\left[1-\frac{2 m}{(m+1)}\left(\frac{x_{0}}{\delta}\right)-\frac{m}{(m+2)}\left(\frac{x_{0}}{\delta}\right)^{2}\right], \quad \delta \geq x_{0} .
\end{aligned}
$$

When $\delta \gg x_{0}, \quad(6.8)$ becomes

$$
\left.\delta=t<V^{2}\right\rangle^{1 / 2} .
$$

If a new parameter $\tau_{0}$ is introduced by the relation:

$$
\tau_{0}=x_{0} /\left\langle V^{2}\right\rangle^{1 / 2}
$$


(6.7) and (6.8) are reduced to

$$
\begin{aligned}
\delta & =\left\{\frac{2}{(m+1)(m+2)}\left(\frac{t}{\tau_{0}}\right)^{2}\right\}^{\frac{1}{2-m}}, & & \delta \leqq x_{0}, \\
& =\frac{t}{\tau_{0}} . & & \delta \gg x_{0} .
\end{aligned}
$$

Substitution of (6.10) and (6.6) into (6.4) yields

$$
\boldsymbol{R}_{L}(\xi)=1-\alpha\left(\frac{\xi}{\tau_{0}}\right)^{\frac{2 m}{2-m}}
$$

where

$$
\alpha=\frac{4}{(m+1)(2-m)^{2}}\left\{\frac{2}{(m+1)(m+2)}\right\}^{\frac{m}{2-m}}
$$

The equations (6.5), (6.6) and (6.11) show clearly the relationship among the Taylor-Kármánian, Eulerian and Lagrangian correlation functions.

When $\boldsymbol{R}_{T \kappa}(x)$ is given by

$$
\boldsymbol{R}_{T K}(x)=1-\left(\frac{x}{x_{0}}\right)^{2 / 3},
$$

as is widely accepted in the recent theory on the isotropic turbulence, we get

$$
\begin{aligned}
& \boldsymbol{R}_{\theta}(f)=1-\left(\frac{t}{t_{0}}\right)^{2 / 3}, \\
& \boldsymbol{R}_{L}(\xi)=1-\alpha\left(\frac{\xi}{\tau_{0}}\right)
\end{aligned}
$$

The equation (6.14) was once proposed by Kampe de Feriet (1939) and was recently again derived theoretically by taking account of the similarity theory of turbulence.

The equation (6.14) shows that the value of $R_{L}(\xi)$ vanishes, when $\xi=\tau_{0} / \alpha$. From this physical meaning, $\tau_{0}$ defined by $(6.9)$ may be reasonably called the "life time" of the largest turbulent element.

$\tau_{0}$ in (6.9) corresponds to "Abfallszeit" in weizsäcker's paper (1948), i. e.; he introduced the "Abfallszeit" $\left(\tau_{n}\right)$ of the $n$-rank turbulent element by the relation

$$
\tau_{n}=\frac{L_{n}}{V_{n}} \text {. }
$$

and gave the explanation of the physical meaning of $\tau_{n}$ as follows: " $\tau_{n}$ ist diejenige Zeit, in der sich das Aussehen der Strömung in Gebieten der Grössenordnung $\mathrm{L}_{n}$ merklich verändert". In other words, since the rate of dissipation of energy per unit time and unit mass of the fluid is proportional to $V_{n}{ }^{3} / L_{n}$ and the kinetic energy of the turbulent element of size $L_{n}$ is proportional to $V_{n}^{2}$, the energy is dissipated completely after time

$$
\begin{aligned}
V_{n}^{2} /\left(\frac{V_{n}^{3}}{L_{n}}\right)= & \frac{L_{n}}{V_{n}}=\tau_{n} . \\
& -12-
\end{aligned}
$$


F. N. Frenkiel (1952 a, b) used in his investigations on the turbulent diffusion the "Lagrangian scale of turbulence" defined by

$$
L_{h}=\int_{0}^{\infty} R(\xi) d \xi
$$

As can be seen easily from (6.14), $L_{h}$ thus defined is closely connected with the life time.

In any way, it is to be stressed that the characteristic time scale in $\boldsymbol{R}_{B}(t)$ is the passage time and that in $\boldsymbol{R}_{L}(\xi)$ is the life time of the largest turbulent element in that turbulent field.

We shall now show briefly, following. O. G. Setton's procedure (1949), that the logarithmic law of the wind profile near the ground surface can be derived, when the Lagrangian correlation is given by (6.11).

As to the vertical transfer of momentum in the lowest layer of the atmophere, $\tau_{0}$ of the vertical largest turbulent element is of the order of several seconds and the condition $t \gg \tau_{0}$ is satisfied after a relatively short time.

Therefore, the most effective turbulent element which determines the stationary vertical wind profile is the largest one in that turbulent field and it is needed to confine our attention to that largest turbulent element. Then, the turbulent viscosity $K$ is given by

$$
\begin{aligned}
K & =\left\langle W^{2}>\int_{0}^{\infty} R_{L}(\xi) d \xi=\left\langle W^{2}>\int_{0}^{\tau_{0} \alpha^{-\frac{L}{\beta}}}\left\{1-\alpha\left(\frac{\xi}{\tau_{0}}\right)^{\beta}\right\} d \xi\right.\right. \\
& =\left\langle W^{2}>\tau_{0} \alpha^{-\frac{1}{\beta}} \frac{\beta}{\beta+1},\right.
\end{aligned}
$$

where $\beta=\frac{2 m}{2-m} \quad \tau_{0}$ in (6.15) should be given by $\tau_{0}=l /\langle W\rangle^{1 / 2}$, where $l$ is the mixing length.

$K$ is therefore given by

$$
K \sim<W^{2}>^{1 / 2} l
$$

If the frequency distribution of the velocities are assumed to follow the Maxwellian law of distribution,

$$
\left.\left\langle W^{2}\right\rangle=\frac{1}{2} \pi<|W|\right\rangle^{2} .
$$

Following Prandtl, we assume

$$
\left\langle|W|>=l-\frac{\partial U}{\partial z}\right.
$$

where $U$ denotes the mean velocity at level $z$. Then

$$
\left\langle W^{3}\right\rangle=\frac{1}{2} \pi l^{2}\left(\frac{\partial U}{\partial z}\right)^{2}
$$


On the other hand, following Th. v. Kármán, we assume that $l$ is given by

$$
l=k_{0} \frac{\partial U}{\partial z} / \frac{\partial^{2} U}{\partial z^{2}}
$$

Then, from $(6.15) \sim(6.18)$, we get

$$
\left.K \sim l<W^{2}\right\rangle^{1 / 2}=\sqrt{\frac{\pi}{2}} l^{2}\left(\frac{\partial U}{\partial z}\right)=\sqrt{\frac{\pi}{2}} k_{0}\left(\frac{\partial U}{\partial z}\right)^{3} /\left(\frac{\partial^{2} U}{\partial z^{2}}\right)^{2}
$$

In the lowest layer of the atmosphere, it is reasanably assumed that the turbulent stress does not vary with height, i.e.,

$$
K \frac{\partial U}{\partial z}=\text { const. }
$$

From (6.19) and (6.20), we get

$$
\left(\frac{\partial U}{\partial z}\right)^{4} /\left(\frac{\partial^{2} U}{\partial z^{2}}\right)^{2}=\text { const. }
$$

The solution of $(6.21)$ is

$$
\frac{\partial U}{\partial z}=\frac{\text { const }}{z}, \quad \text { or } \quad U \sim \log z .
$$

We get thus the well-known logarithmic law of the wind profile. It is note worthy, however, that this law is derived, irrespective of the value of $m$ in the Lagrangian correlation.

In conclusion, the authors wish to express their hearty thanks to Rrof. S. Syono for his kind encouragement and guidance.

\section{References}

Batchelor, G. K., 1950: The Application of the Similarity Theory of Turbulence to Atmopheric Diffusion. Quart. Journ. Roy. Met. Soc. 76, pp. 133-146.

Frenkiel, F. N., 1946: On Turbulent Diffusion. Proc. U. S. Naval Ord. Lab. Res. Symposia.

1952 a: On the Statistical Theory of Turbulent Diffusion. Proc. Nat. Acad. Sci. 38, pp. 509-515.

b : Application of the Statistical Theory of Turbulent Diffusion to Micro-meteorology Journ. Met. 9, pp. 252-259.

Ichiye, T., 1951: Theory of Oceanic Turbulence. Ocean. Mag. 3, pp. 82-87.

Inoue, E., 1950: On the Structure of the Wind near the Ground (I). Journ. Met. Soc. Japan, 28

1951: On the Turbulent Diffusion in the Atmosphere (II). Ibid. 29 pp. 32-39.
1952: On the Structure of Wind near the Ground. (In Japanese.) Bull. Nat. Inst. Agr. Sci. Ser. A, No. 2.

Ogura, Y., 1952 a: The Theory of Turbulent Diffusion in the Atmosphere (I). Journ. Met. Soc.. Japan, 30 pp. 23-28.

___ b: The Theory of Turbulent Diffusion in the Atmosphere (II). Ibid. pp. 53-58.

c: The Theory of Turbulent Diffusion in the Atmosphere (III). Ibid. pp. 386-397. and K. Miyakoda, 1953: A paper to be published. 
Proudman, J., 1948: On the Mixing of Sea Water by Turbulence. Proc. Roy. Soc. A, 195, pp. 300-309.

Sutton, O. G., 1949: Atmospheric Turbulence: Methuen, London.

Weizsäcker, C. F. von, 1948: Das Spectrum der Turbulenz bei grossen Reynoldschen Zahlen. Z. f. Phys. 124, ss. 614-627. 